\title{
Simultaneous Chandra and VLA Observations of the Transitional Millisecond Pulsar PSR J1023+0038: Anti-correlated X-Ray and Radio Variability
}

\author{
Slavko Bogdanov $^{1}$ (iD), Adam T. Deller ${ }^{2}$ (D), James C. A. Miller-Jones ${ }^{3}$ (D), Anne M. Archibald ${ }^{4,5}$ (iD), Jason W. T. Hessels ${ }^{4,5}$ (D), \\ Amruta Jaodand $^{4,5}$ (D), Alessandro Patruno ${ }^{6}$ (D) , Cees Bassa $^{5}$, and Caroline D’Angelo ${ }^{6}$ \\ ${ }^{1}$ Columbia Astrophysics Laboratory, Columbia University, 550 West 120th Street, New York, NY 10027, USA \\ ${ }^{2}$ Centre for Astrophysics and Supercomputing, Swinburne University of Technology, Hawthorn, VIC 3122, Australia \\ ${ }^{3}$ International Centre for Radio Astronomy Research-Curtin University, GPO Box U1987, Perth, WA 6845, Australia \\ ${ }^{4}$ Anton Pannekoek Institute for Astronomy, University of Amsterdam, Science Park 904, NL-1098 XH Amsterdam, The Netherlands \\ 5 ASTRON, Netherlands Institute for Radio Astronomy, Postbus 2, NL-7990 AA Dwingeloo, The Netherlands \\ ${ }^{6}$ Leiden Observatory, Leiden University, P.O. Box 9513, NL-2300 RA Leiden, The Netherlands \\ Received 2017 September 25; revised 2018 February 8; accepted 2018 February 8; published 2018 March 23
}

\begin{abstract}
We present coordinated Chandra X-ray Observatory and Karl G. Jansky Very Large Array observations of the transitional millisecond pulsar PSR J1023+0038 in its low-luminosity accreting state. The unprecedented five hours of strictly simultaneous X-ray and radio continuum coverage for the first time unambiguously show a highly reproducible, anti-correlated variability pattern. The characteristic switches from the X-ray high mode into a low mode are always accompanied by a radio brightening with a duration that closely matches the X-ray low mode interval. This behavior cannot be explained by a canonical inflow/outflow accretion model where the radiated emission and the jet luminosity are powered by, and positively correlated with, the available accretion energy. We interpret this phenomenology as alternating episodes of low-level accretion onto the neutron star during the X-ray high mode that are interrupted by rapid ejections of plasma by the active rotation-powered pulsar, possibly initiated by a reconfiguration of the pulsar magnetosphere, that cause a transition to a less X-ray luminous mode. The observed anti-correlation between radio and X-ray luminosity has an additional consequence: transitional MSPs can make excursions into a region of the radio/X-ray luminosity plane previously thought to be occupied solely by black hole X-ray binary sources. This complicates the use of this luminosity relation for identifying candidate black holes, suggesting the need for additional discriminants when attempting to establish the true nature of the accretor.
\end{abstract}

Key words: accretion, accretion disks - pulsars: individual (PSR J1023+0038) - X-rays: binaries

\section{Introduction}

PSR J1023+0038 is an archetypal "missing link" binary millisecond pulsar (Archibald et al. 2009). The binary system contains a $P=1.69 \mathrm{~ms}$ pulsar and a $0.2-0.5 M_{\odot}$ secondary star (Deller et al. 2012; McConnell et al. 2015) in a $4.8 \mathrm{hr}$ orbit. It is the first system to show evidence of alternating radio-loud pulsar and X-ray binary states (Thorstensen \& Armstrong 2005; Wang et al. 2009). The system underwent a transformation to a low-luminosity accreting state in 2013 June (Patruno et al. 2014; Stappers et al. 2014), in which it has remained until present.

In the low-luminosity accreting state, PSR J1023+0038 exhibits puzzling X-ray modulation characterized by rapid switching between two clearly distinguishable luminosity levels (referred to as "modes") of $\approx 3 \times 10^{33}$ and $\approx 5 \times 10^{32} \mathrm{erg} \mathrm{s}^{-1}$ (0.3-10 keV), plus sporadic flares (Tendulkar et al. 2014; Bogdanov et al. 2015). This unusual large-amplitude variability appears to be a generic feature of accreting transitional millisecond pulsars (tMSPs; see de Martino et al. 2013; Linares et al. 2014; Bogdanov \& Halpern 2015). XMM-Newton observations also revealed coherent X-ray pulsations at the $1.7 \mathrm{~ms}$ pulsar rotation period known from the radio pulsar state (Archibald et al. 2015), which strongly suggest active accretion at very low luminosities $\left(\sim 10^{33} \mathrm{erg} \mathrm{s}^{-1}\right)$. This discovery comes as a surprise because the implied accretion rate $\left(\sim 10^{-5}-10^{-4}\right.$ times the Eddington luminosity) is expected to be too low to overcome the "centrifugal barrier" imposed by the pulsar's $\sim 10^{8} \mathrm{G}$ magnetic field and rapid spin. Unlike luminous accreting millisecond X-ray pulsars (AMXPs; see Patruno \& Watts 2012, for a comprehensive overview), the pulsations are only observed in the steady "high" mode (in which the system spends $\sim 75 \%$ of its time) and they seem to switch off during the "low" mode $(20 \%$ of the time) and in the bright flares (1\%-2\% of the time). Furthermore, the X-ray high mode flux and pulse shape are so stable that they are nearly identical in observations spaced years apart (Bogdanov et al. 2015; Jaodand et al. 2016). The presence of channeled accretion at a very specific accretion rate and the peculiar X-ray flux bimodality are very puzzling and cannot be satisfactorily explained by existing accretion models.

A long-term X-ray timing study with XMM-Newton (Jaodand et al. 2016) revealed that, in its accreting state, PSR $\mathrm{J} 1023+0038$ is spinning down $26.8 \pm 0.4 \%$ faster compared to its previous disk-free radio pulsar state. The implication of this finding is that accretion does not dramatically alter the pulsar spin-down rate in this state, and that the pulsar wind is still active at a similar (albeit potentially not identical) level as in the disk-free rotation-powered state.

At radio frequencies, observations have shown that in its accreting state PSR $\mathrm{J} 1023+0038$ is not detectable as a radio pulsar (Stappers et al. 2014). However, Very Large Array (VLA) radio imaging during this state revealed rapidly variable (with factor-of-two variability within minutes and order-ofmagnitude variability on timescales of $\sim 30$ minutes) flatspectrum emission that persisted over a period of at least 6 months (Deller et al. 2015). Using near-simultaneous radio and X-ray observations, Deller et al. (2015) found that this emission is consistent with a compact, partially self-absorbed synchrotron jet, and that PSR J1023+0038 (and other tMSPs) 
exhibit much stronger jets than predictions based on observations of neutron stars accreting at much higher rates.

At present, it is not clear if/how the radio variability is causally related to the X-ray behavior. In particular, the jet production mechanism might operate in only one or two of these X-ray modes; alternatively, a jet may be present in all modes but with substantially changing spectral and temporal properties. Previous coordinated X-ray and radio observations only resulted in a brief interval of simultaneous coverage, which proved inconclusive in establishing any correlated behavior between the two wavelength bands (Bogdanov et al. 2015).

In this paper, we present an analysis of strictly simultaneous X-ray and radio continuum observations of PSR J1023+0038 during the current accreting state. The results provide important new information regarding the puzzling X-ray and radio behavior, and their relation. The work is organized as follows. In Section 2 we discuss the X-ray and radio observations and the data reduction procedures. In Section 3 we present a variability and correlation analysis of the X-ray and radio time series. In Section 4, we focus on the X-ray/radio luminosity relation in light of the new results for PSR J1023+0038. We discuss the implications of the results in Section 5 and offer concluding remarks in Section 6.

\section{Observations and Data Analysis}

\subsection{Chandra X-Ray Observatory}

PSR J1023+0038 was observed with Chandra as part of the Cycle 17 Guest Observer program (ObsID 17785) on 2016 October 21 for a duration of $20 \mathrm{ks}$. The back-illuminated ACISS3 chip was placed at the aim point. To avoid photon pile-up ${ }^{7}$ that would be caused by the high source count rate, the detector was used in Continuous Clocking (CC33_GRADED) mode, which enables a rapid $(2.85 \mathrm{~ms})$ readout time by sacrificing one dimension of spatial imaging.

The data analysis was carried out using $\mathrm{CIAO}^{8}$ version 4.9 (Fruscione et al. 2006) and the accompanying calibration database CALDB 4.7.3. Following recommended procedures, the source and background events were extracted from rectangular regions of width $5^{\prime \prime}$ along the imaging direction. Due to the bright nature of PSR J1023+0038, the background contribution to the source count rate is negligible.

The event timestamps were translated from the Terrestrial Time (TT) standard used by Chandra to UTC in order to enable alignment with the radio light curve. As the readout time of the Chandra data is greater than the $1.69 \mathrm{~ms}$ pulsar spin period, a pulsation/timing analysis is not possible.

\subsection{Very Large Array}

A five-hour observation with the Karl G. Jansky Very Large Array (VLA) was performed on 2016 October 21 from UT 11:16 to 16:15, entirely overlapping with the Chandra observation. The array was in the A configuration and the observational setup and calibration were identical to those described in Deller et al. (2015), sampling a $4 \mathrm{GHz}$ bandwidth from 8 to $12 \mathrm{GHz}$, using $3 \mathrm{C} 286$ as the primary flux density

\footnotetext{
7 Event pile-up in CCDs occurs when two or more events fall on a detector pixel during the same readout frame. As a consequence, the intrinsic source spectrum is distorted and degraded. This deleterious effect can be mitigated by increasing the readout rate.

8 Chandra Interactive Analysis of Observations.
}

calibrator, and using J1024-0052 as the gain calibrator with a cycle time of 8 minutes. The VLA pipeline in CASA version 4.5.0 (McMullin et al. 2007) was used for initial calibration, followed by a small amount of additional flagging on the target field and self-calibration using the field model described in Deller et al. (2015). The self-calibration procedure included the peeling (subtraction from the visibilities after directiondependent calibration) of the brightest background source in the field.

Imaging was performed in an automated fashion in small windows that surrounded PSR J1023+0038 and several other background sources in the field, with selection criteria applied to produce a time series binned at 30 and $60 \mathrm{~s}$ time resolutions over the entire observation, using the full frequency bandwidth.

In addition, we produced a number of stacked data sets that give higher sensitivities and hence allow a more detailed analysis of flux density and spectral index variations. For these analyses, we produced single-frequency and dual-frequency (8-10 and 10-12 GHz) data sets for plotting, and also data sets with five equally spaced frequency segments to better estimate the spectral index and its error. First, we stacked all the time ranges corresponding to (1) clear X-ray high modes, and (2) clear X-ray low modes. We stacked the $\sim 4$-minute intervals that correspond to the rising and decaying phase of the radio flare, which follows the sole bright X-ray flare at UT 12:40. Finally, we stacked time ranges corresponding to X-ray low modes of similar duration, using the high $\rightarrow$ low mode transition as a reference point for alignment. This allowed us to compare long (duration $~ 7.7$ minutes), medium (duration $\sim 3.3$ minutes), and short (duration $\sim 1.5$ minutes) low modes.

The source flux density was extracted using the CASA task imfit with a starting model fixed at the known source position, while the image noise was estimated using an emission-free region of the image offset by several arcseconds from the source. Failed fits and non-detections were replaced by upper limits at twice the image noise.

\subsection{Archival Data}

In Section 3, we revisit the contemporaneous XMM-Newton and VLA observations from 2013 November 10 originally presented in Bogdanov et al. (2015) and Deller et al. (2015; see those papers for the details of the observations and data reduction). In previous analyses of the XMM-Newton data, we only considered the European Photon Imaging Camera (EPIC) MOS1/2 and PN instruments, which overlapped for 16 minutes with the VLA observation. However, due to a shorter instrumental setup time, the Reflection Grating Spectrometer (RGS) exposure begins 30 minutes earlier, providing a longer interval of simultaneity with the radio time series.

We used the XMM-Newton Science Analysis System ${ }^{9}$ (SAS; Gabriel et al. 2004) version xmmsas_20170112_133716.0 .0 to process the RGS data. A combined RGS1 and RGS2, first- and second-order, exposure-corrected, and background-subtracted light curve binned at 20 seconds was produced using the rgslccorr tool in SAS.

\footnotetext{
9 The XMM-Newton SAS is developed and maintained by the Science Operations Centre at the European Space Astronomy Centre and the Survey Science Centre at the University of Leicester.
} 


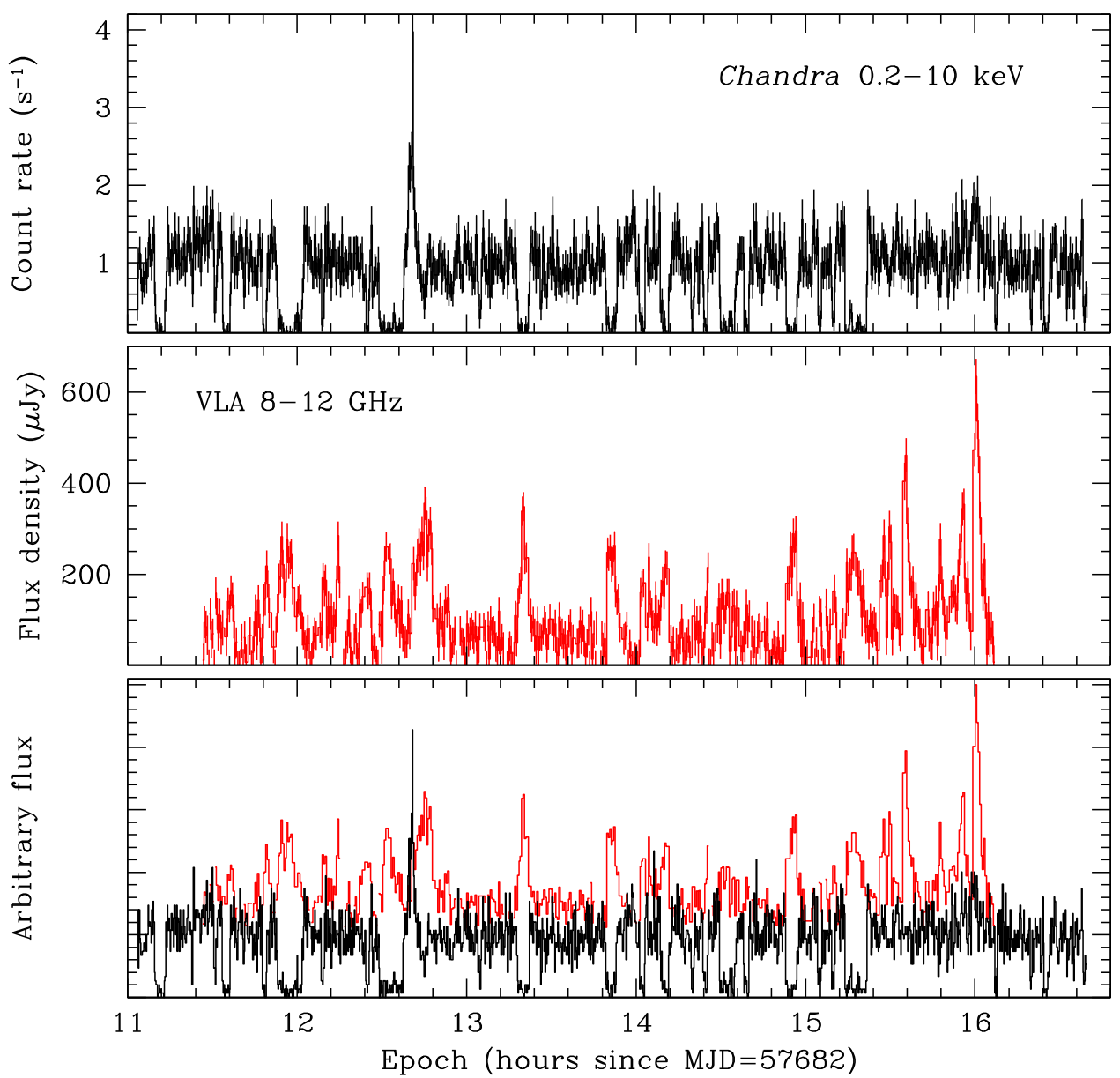

Figure 1. Top: Chandra $0.2-10 \mathrm{keV}$ (black) exposure-corrected and background-subtracted count rate time series of PSR J1023+0038 grouped in $25 \mathrm{~s}$ intervals. The data show the three distinct X-ray flux modes (low, high, and flare) seen in previous observations. Middle: VLA 8-12 GHz light curve binned at a $30 \mathrm{~s}$ resolution. Bottom: the same light curves from the upper panels superposed, with the error bars omitted to improve clarity. The strong anti-correlation, characterized by the increased radio activity associated with each X-ray low mode, is obvious.

\section{Variability Analysis}

The joint Chandra/VLA observations resulted in $\sim 5 \mathrm{hr}$ of strictly simultaneous coverage. The acquired exposure-corrected $\mathrm{X}$-ray light curve in the $0.2-10 \mathrm{keV}$ range binned in $25 \mathrm{~s}$ intervals and the radio light curve in the $8-12 \mathrm{GHz}$ band with a $30 \mathrm{~s}$ time resolution are shown in Figure 1. The bin sizes were chosen to provide an optimal balance between signal-to-noise ratio $(\mathrm{S} / \mathrm{N})$ and time resolution. The $\mathrm{X}$-ray light curve exhibits the characteristic variability pattern seen in previous observations (Patruno et al. 2014; Stappers et al. 2014; Bogdanov et al. 2015; Jaodand et al. 2016), namely, rapid and aperiodic switching between two distinct flux levels at $\approx 5 \times 10^{32}$ (the low mode) and $\approx 3 \times 10^{33} \mathrm{erg} \mathrm{s}^{-1}$ (the high mode), plus a short-lived flaring episode at UT 12:40 reaching $\sim 10^{34} \mathrm{erg} \mathrm{s}^{-1}$.

The VLA time series shows numerous rapid flares that generally last a few minutes, interspersed between periods of relatively steady faint emission. A striking anti-correlated variability pattern between the X-ray and radio is immediately apparent. Nearly every X-ray low mode interval is accompanied by a temporally coincident radio brightening, while the steady low-level radio emission occurs during the X-ray high modes. The "quiescent" radio flux during the X-ray high modes has an average flux density at the reference frequency of $10 \mathrm{GHz}$ of $S_{\nu}=56 \pm 2 \mu \mathrm{Jy}$ and a spectral index of $\alpha=0.2 \pm 0.2$ (where $\left.S_{\nu} \propto \nu^{\alpha}\right)$. A delayed radio flare follows the brief but intense X-ray flare around UT 12:40, with an average radio flux density at $10 \mathrm{GHz}$ of $235 \pm 9 \mu \mathrm{Jy}$.

Apart from the radio brightening seen during X-ray low modes, and following the one well-defined X-ray flare, several other radio flares that are not clearly associated with any particular X-ray activity are seen, mostly during the time window UT $\sim 15: 30$ to 16:00. The peak radio luminosity over the entire observation is actually attained during one of these "unassociated" radio flares. Some weak X-ray flaring appears to be present around UT 15:50 to 16:00 (see Figure 1), but several of the radio flares with no X-ray counterparts occur prior to this window of weak X-ray flaring activity.

As expected from a visual inspection of the light curves, a scatterplot of the overlapping X-ray and radio time series shown in Figure 2 (with the Chandra data rebinned to match the VLA time bins) shows two distinct clusters of points for the high (blue) and low (red) X-ray modes, with the low mode points clustered around a systematically higher radio luminosity. The archival XMM-Newton RGS and VLA data from 2013 November 10 show the same anti-correlation for the two low mode intervals covered in the $\sim 45$-minute segment with simultaneous data (see Figure 3), though due to the brief overlap, the results were previously inconclusive when considering this data set in isolation.

One of the remarkable features of the variability is that the rise and fall of the radio luminosity seems to very closely match 


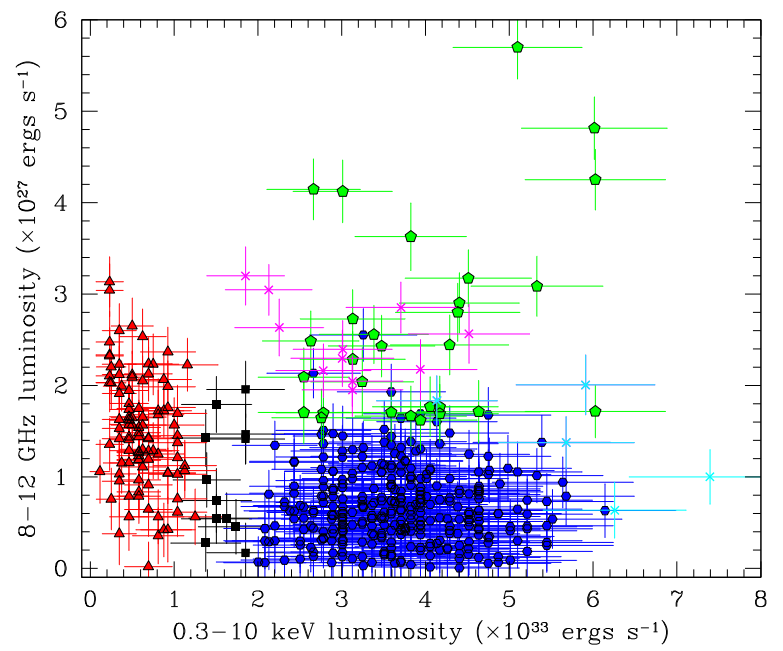

Figure 2. A scatterplot of $8-12 \mathrm{GHz}$ radio luminosity (in units of $10^{27} \mathrm{erg} \mathrm{s}^{-1}$ ) vs. $0.3-10 \mathrm{keV}$ X-ray luminosity (in units of $10^{33} \mathrm{erg} \mathrm{s}^{-1}$ ) for the strictly simultaneous VLA and Chandra data shown in Figure 1. The blue circles and red triangles mark the high and low X-ray modes, respectively, while the black crosses correspond to the ambiguous points that lie between the two modes. The green squares show the "unassociated" radio flares seen toward the end of the observation, the cyan stars are the time bins during the bright X-ray flare, and the magenta stars are the time bins during the subsequent delayed radio flare.

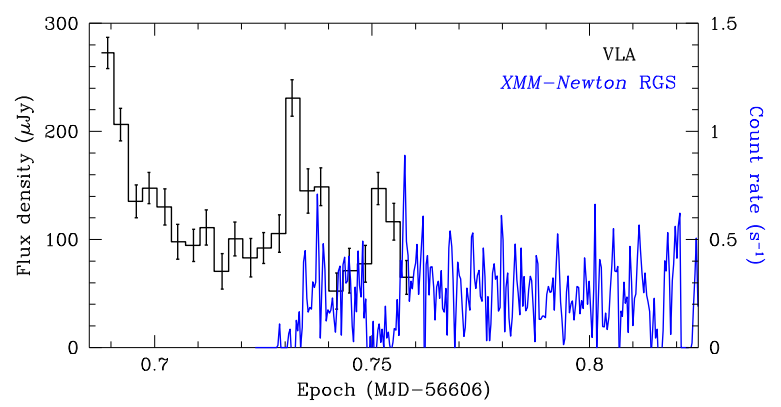

Figure 3. Contemporaneous VLA $5.9 \mathrm{GHz}$ (black) and XMM-Newton RGS 0.3-10 keV (blue) time series of PSR J1023+0038 obtained on 2013 November 10. The anti-correlated variability is evident, with a significant increase in radio flux during the two X-ray low modes that occur during the overlap.

the transitions to and from the X-ray low mode. Specifically, the radio brightening associated with the shorter duration low modes has correspondingly faster rise and decay times. Furthermore, there does not appear to be a strong dependence of the peak radio luminosity on the duration of a low mode; some of the short- and medium-duration low mode intervals reach a radio luminosity that is comparable to that of the longest low modes (see Figure 4).

\subsection{Time-resolved Spectral Variations}

As found in previous studies (Bogdanov et al. 2015; Campana et al. 2016), the X-ray spectrum of PSR J1023 +0038 only undergoes a modest change between the three modes. The spectrum is power-law-like, with spectral photon index $\Gamma \approx 1.7$ at all luminosity levels.

Considering the average radio spectral index for PSR J1023 +0038 , as shown in Table 1, suggests similarly minor variations between $\mathrm{X}$-ray modes: $\alpha=0.2 \pm 0.2$ in the high mode, $\alpha=-0.1 \pm 0.2$ averaged across all low modes, and $\alpha=0.3 \pm 0.4$ during the radio flare following the prominent
X-ray flare at UT 12:40. However, averaging masks considerable variation during each radio brightening associated with an $\mathrm{X}$-ray low mode. While the sensitivity of the VLA data is insufficient to resolve each individual low mode both spectrally and temporally, stacking allows us to investigate the average radio spectral evolution during a low mode. As shown in Table 1, the radio spectrum evolves from significantly inverted ( $\alpha \simeq 0.4$ ) to relatively steep $(\alpha \simeq-0.5$ ) over the course of several minutes during a typical low mode.

As noted previously, the one prominent X-ray flare in the Chandra time series lasting $\sim 2$ minutes at UT 12:40 is followed by a delayed and longer-lasting radio flare (labeled F1 in Figure 4). Since we only have one such flare and cannot stack radio data as we can for the low modes, our sensitivity is too limited to show a significant variation in the spectral index over the course of the flare. When the radio flare is divided into a rising phase (UT 12:40:48-12:44:48) and decaying phase (UT 12:44:48-12:48:36), the spectral index is $\alpha=0.4 \pm 0.5$ in the rising phase, and $\alpha=0.1 \pm 0.5$ in the decaying phase. While this is consistent with a transition from inverted to steep (as is seen in the X-ray low modes, and would be expected for an expanding region of synchrotron-emitting plasma), it is also consistent with a constant spectral index (as is seen in, e.g., the $\mathrm{X}$-ray high mode).

\subsection{Comparison of X-Ray Low Modes as a Function of Duration}

Although the individual low mode radio brightening events do not have sufficient $\mathrm{S} / \mathrm{Ns}$ to determine the radio spectral evolution, by combining multiple intervals it is possible to place informative constraints. From the 23 low mode intervals identified in the X-ray data, 18 have strictly simultaneous VLA coverage. Seven of these have a duration of less than $60 \mathrm{~s}$, making them very difficult to resolve with our $30 \mathrm{~s}$ radio data, leaving 11 low mode intervals with durations 75-500 s. For the purposes of investigating the effects of low mode duration on the radio emission, we divide these into three bins: $88 \pm 12 \mathrm{~s}$, $200 \pm 15 \mathrm{~s}$, and $460 \pm 40 \mathrm{~s}$. Two low modes, lasting $126 \mathrm{~s}$ and $277 \mathrm{~s}$, could not be included in any bin without broadening the bin too far, and were discarded from the following analysis. For each bin (long, medium, and short) we stack the three included individual low modes, using the high $\rightarrow$ low mode transition boundary as a reference point. The results are shown in Figure 4, with the L, M, and S labels assigned to the long, medium, and short flares, respectively. Finally, to highlight the spectral evolution during outbursts, we reproduce the stacked images shown in the right column of Figure 4 with the upper and lower halves of the observed frequency range (8-10 and 10-12 GHz) plotted separately, reducing the temporal resolution to $60 \mathrm{~s}$ to maintain sensitivity (see Figure 5). We discuss the findings from the analysis of these light curves in Section 5.

\section{Revisiting the X-Ray Binary Radio-X-Ray Luminosity Relation}

Black hole (BH) low-mass X-ray binaries (LMXBs) exhibit a well-defined relation between their $X$-ray and radio luminosities of $L_{R} \propto L_{X}^{0.61}$ (Gallo et al. 2014). Neutron stars (NSs) at high luminosities $L_{X} \gtrsim 10^{36} \mathrm{erg} \mathrm{s}^{-1}$, on the other hand, may follow two possible correlations, $L_{R} \propto L_{X}^{0.7}$ or $L_{R} \propto L_{X}^{1.4}$, depending on whether they are in a radiatively efficient or inefficient regime (Migliari \& Fender 2006). X-ray 

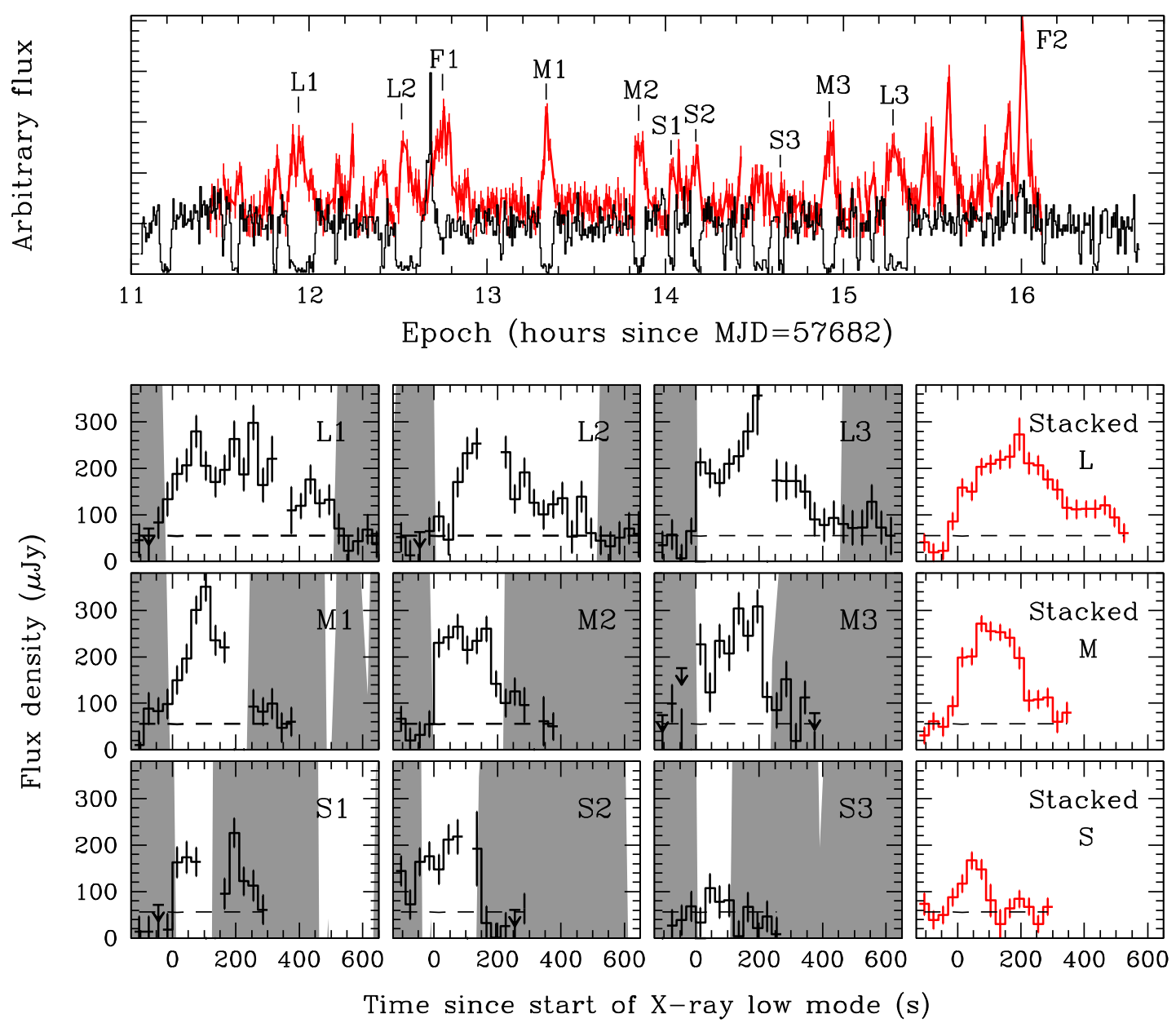

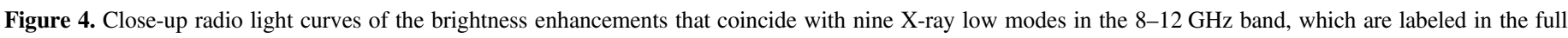

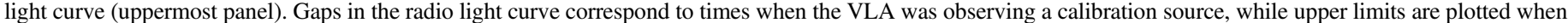

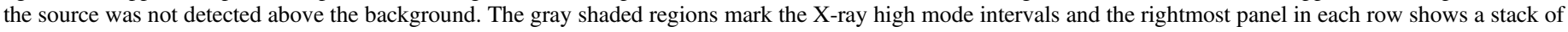
the three low mode light curves of comparable duration.

Table 1

Radio Flux Density and Spectral Index of PSR J1023+0038

\begin{tabular}{lcr}
\hline \hline Selection Criterion & $S_{10 \mathrm{GHz}}(\mu \mathrm{Jy})$ & Spectral Index $\alpha$ \\
\hline X-ray high mode (all) & $56 \pm 2$ & $0.2 \pm 0.2$ \\
X-ray low mode (all) & $168 \pm 4$ & $-0.1 \pm 0.2$ \\
X-ray low mode (1st half) & $196 \pm 6$ & $0.4 \pm 0.3$ \\
X-ray low mode (2nd half) & $156 \pm 6$ & $-0.5 \pm 0.3$ \\
Radio flare F1 (all) & $235 \pm 9$ & $0.3 \pm 0.4$ \\
Radio flare F1 (1st half) & $224 \pm 13$ & $0.4 \pm 0.5$ \\
Radio flare F1 (2nd half) & $260 \pm 11$ & $0.1 \pm 0.5$ \\
\hline
\end{tabular}

and radio flux measurements obtained for the three confirmed accreting transitional MSPs, PSR J1023+0038 (Deller et al. 2015), XSS J12270-4859 (Hill et al. 2011), and J1824 -2452I (Papitto et al. 2013), have established that X-ray binaries containing MSPs, ${ }^{10}$ can have much higher radio luminosities than predicted from extrapolating the $L_{X}-L_{R}$ relation for high accretion rate neutron star LMXBs from a limited range of $L_{X}$ (Migliari et al. 2011). At $L_{X} \lesssim 10^{34} \mathrm{erg} \mathrm{s}^{-1}$,

\footnotetext{
${ }^{10}$ The tMSP candidate 1RXS J154439.4-112820 (3FGL J1544.6-1125; Bogdanov \& Halpern 2015) falls in the same region as the confirmed tMSPs (A. Jadoand et al. 2018, in preparation).
}

the time-averaged radio luminosities of tMSPs are less than an order of magnitude below the $\mathrm{BH}$ relation.

Figure 6 shows the $L_{X}-L_{R}$ plane for NS and BH LMXB systems, including recent measurements for EXO 1745-248 (Tetarenko et al. 2016), 1RXS J180408.9-342058 (Gusinskaia et al. 2017), three AMXPs plus Cen X-4 (Tudor et al. 2017), and the BH LMXB candidates in the globular clusters M22 (Strader et al. 2012), M62 (Chomiuk et al. 2013), and 47 Tuc (Miller-Jones et al. 2015; Bahramian et al. 2017).We also add points for PSR J1023+0038 corresponding to the high and low modes from the simultaneous Chandra and VLA observations presented here, as well as the long-term median $L_{X}(1-10 \mathrm{keV})$ and $L_{R}(5 \mathrm{GHz})$ values obtained from the 10 VLA and Swift observations presented in Deller et al. (2015). All luminosities for PSR J1023+0038 were computed assuming the parallax distance of $1.368_{-0.039}^{+0.042} \mathrm{pc}$ (Deller et al. 2012). The $1-10 \mathrm{keV}$ $\mathrm{X}$-ray luminosities for the high, low, and median cases are $2.4 \times 10^{33}, 4.0 \times 10^{32}$, and $1.9 \times 10^{33} \mathrm{erg} \mathrm{s}^{-1}$, while the corresponding $5 \mathrm{GHz}$ radio luminosities are $6 \times 10^{26}$, $2 \times 10^{27}$, and $1 \times 10^{27} \mathrm{erg} \mathrm{s}^{-1}$. An important consequence of the anti-correlated behavior of PSR J1023+0038 is that, in the $\mathrm{X}$-ray/radio luminosity plane, the system makes excursions close to the black hole X-ray binary relation.

While PSR J1023+0038 spends only 20\% of the time in the X-ray low mode, the duty cycle of this mode in other 


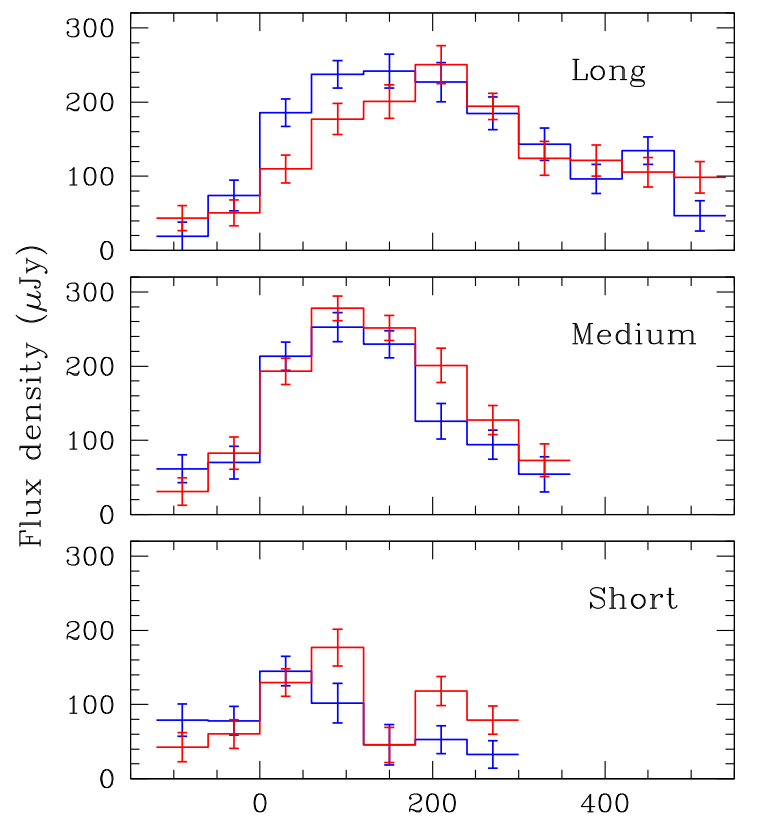

Time since start of $X$-ray $\mathbb{~ l o w ~ m o d e ~ ( s ) ~}$

Figure 5. Top panel: stacked radio light curves of the flares that coincide with the three longest X-ray low modes (labeled L1 through L3 in Figure 4) in the 8-10 GHz (red) and $10-12 \mathrm{GHz}$ (blue) bands. Middle panel: stacked radio light curves for the three low modes of duration 200 s (labeled M1 through M3 in Figure 4). Bottom panel: stacked radio light curves of the three shortest X-ray low modes (labeled S1 through S3 in Figure 4). For all three cases, the zero-time reference corresponds to the start of the X-ray low mode interval.

tMSPs can be significantly higher. For instance, Chandra observations of the tMSP PSR J1824-2452I from 2008 showed that this system can reside in a single low mode interval for $\sim 10 \mathrm{hr}$ (Papitto et al. 2013; Linares et al. 2014). This certainly complicates the use of the $L_{X}-L_{R}$ relation to identify candidate $\mathrm{BHs}$ and invites a close re-examination of globular cluster $\mathrm{BH}$ candidates, for which the ratio of X-ray to radio luminosity obtained from a single short observation is a principal criterion for classifying them as such. The use of radio versus X-ray luminosity as a diagnostic for BH LMXBs could be bolstered when large and rapid variability of the type exhibited by tMSPs can be ruled out, and/or once a significant number of multiepoch observations show consistent results (see Strader et al. 2012; Chomiuk et al. 2013; Bahramian et al. 2017). The anti-correlated X-ray and radio behavior of PSR J1023 +0038 also highlights the necessity for truly simultaneous $\mathrm{X}$-ray and radio observations when attempting to establish the true nature of the accreting compact object.

\section{Discussion}

The simultaneous X-ray/radio data presented here provide valuable new pieces of information on PSR J1023+0038, and by extension, the tMSP class. The most obvious feature, as shown in Figure 1, is the striking anti-correlation of X-ray and radio flux in the high and low modes: the X-ray high modes show X-ray pulsations and low radio luminosity, but when the source switches into its low modes, the X-ray pulsations disappear and the radio emission increases rapidly and dramatically. Figure 4 also shows zoomed-in views of individual low modes, and higher $\mathrm{S} / \mathrm{N}$ stacks of radio light curves during low modes of similar durations. Some common signatures can be discerned: generally, the radio rises quickly, begins to decline before the end of the low mode, and returns to quiescence within 30-60 s of the transition back to the X-ray high mode. Shorter low modes appear to rise faster than longer low modes and can reach comparable peak luminosities, although the shortest flares have consistently fainter peaks. The radio luminosity begins to decline back to the high mode level just before the transition back to the high mode, although in the longest low modes, the radio has already declined to close to the quiescent value. In the shorter low modes on the other hand, the radio emission is still bright and the "cutoff" at the end of the low mode is more apparent.

During the X-ray low modes, there is good evidence that the radio spectrum evolves from being initially inverted to being steep (see Table 1 and Figure 5) -reminiscent of a classical evolving synchrotron spectrum, going from self-absorbed to optically thin on relatively short timescales. This occurs on timescales of several minutes. In contrast, when in the X-ray high mode, the radio emission is normally steady at $\sim 55 \mu \mathrm{Jy}$. However, several instances can be seen in which a radio flare appears to occur during an X-ray high mode with no discernible or only weak enhancement in X-ray emission (see F2 in Figure 4). Finally, the bright X-ray flare event appears to be followed by a radio flare (F1 in Figure 4) that exhibits classic evolving synchrotron features of accretion-driven outflows: a time delay relative to the X-ray event and spectral evolution consistent with expanding plasma (although, as noted, the low signal-to-noise ratio of the radio data also permits a non-evolving spectrum.)

The low radio luminosity during the X-ray high mode, when active accretion onto the star occurs, as evidenced by the coherent X-ray pulsations, can be plausibly interpreted as a weak persistent compact self-absorbed synchrotron jet, commonly observed in both NS (Migliari et al. 2006, 2010) and BH (Fender et al. 2000; Stirling et al. 2001; Gallo et al. 2005) LMXBs. The ejection of a denser discrete plasma cloud can explain the intense X-ray flare and the associated delayed radio flare, which is an expected feature of intermittent jet production (see, e.g., Vadawale et al. 2003; Fender et al. 2004). On the other hand, a similar jet launching scenario seems improbable for the rapid low mode radio brightness increases. In particular, in the conventional accretion/jet production paradigm in X-ray binaries (Blandford \& Payne 1982), the enhancement in accretion rate, observed as increased X-ray flux, is associated with a commensurate increase in jet power, manifested as brighter radio emission in the hard spectral state. However, the anti-correlated X-ray/radio behavior, seen in PSR J1023+0038 at X-ray luminosities characteristic of a hard or quiescent state, implies that a decline in accretion rate is associated with an enhancement instead of a reduction in plasma outflows. Furthermore, a scenario involving radio emission from accretion-driven outflow at large distances from the compact object cannot be reconciled with the fact that the initial rise and decline of the radio flares during the low modes always closely matches the X-ray low mode interval. In particular, once ejected, material in a jet-like outflow no longer maintains causal contact with events occurring near the pulsar, and thus would have no information regarding the X-ray low $\rightarrow$ high mode transition, which is caused by events within the pulsar magnetosphere-inner disk interface. This leads us to conclude that if these radio flares are due to expanding/outflowing synchrotron-emitting plasma (as the spectral evolution 


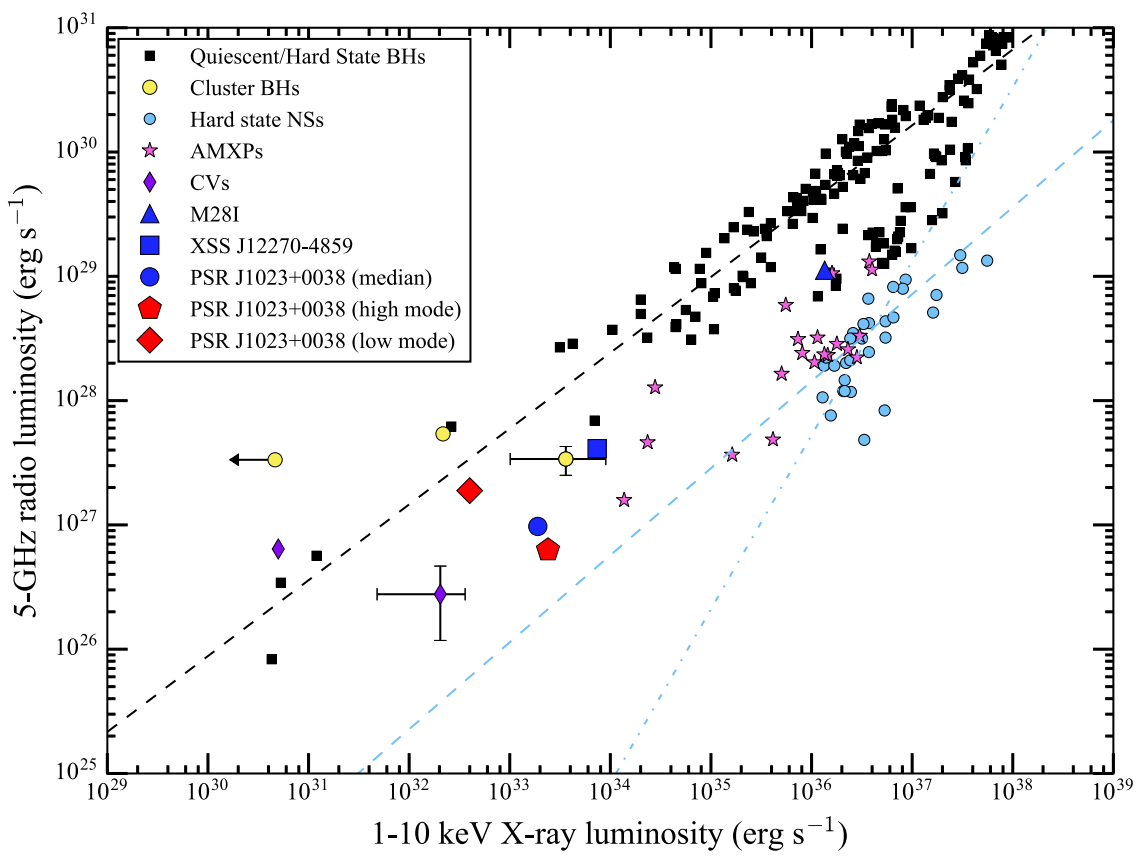

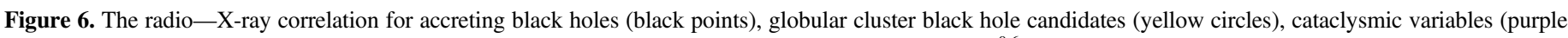

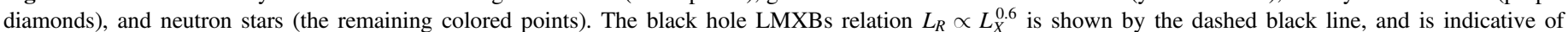

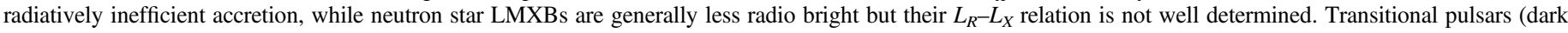

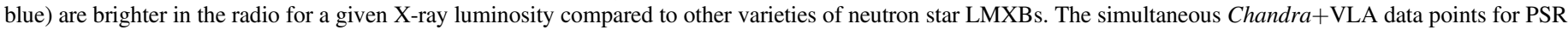
$\mathrm{J} 1023+0038$ (red) show that in the X-ray low mode, this system is indistinguishable from a black hole LMXB.

suggests), the bulk of this emission must arise from plasma in the immediate vicinity of the inner disk region.

The cessation of coherent X-ray pulsations at the high $\rightarrow$ low $\mathrm{X}$-ray mode transition ${ }^{11}$ further implies that the event that leads to the production of the radio flare is also (directly or indirectly) responsible for temporarily disrupting the accretion flow onto the pulsar. Campana et al. (2016) applied a multi-component model, including a radiatively inefficient disk, to the extensive archival XMM-Newton X-ray data of PSR J1023+0038. Based on this, they deduced an inner disk radius of $\sim 21 \mathrm{~km}$ in the $X$-ray high mode and $\sim 200 \mathrm{~km}$ in the X-ray low mode. This finding is consistent with an interpretation in which the accretion flow is pushed out beyond the light cylinder, perhaps by ejection/ expansion of plasma originating in the pulsar magnetosphere, causing a high $\rightarrow$ low X-ray mode transition.

Archibald et al. (2015) and Papitto \& Torres (2015) proposed a basic explanation for the peculiar X-ray modeswitching: in the high modes, magnetically channeled accretion onto the neutron star surface occurs, and when the source switches to a low mode, propeller-mode accretion takes over. This model can account for the anti-correlation we see: in the low modes there is propeller-mode accretion and material is ejected, producing a radio-emitting jet, while in the high modes the material reaches the surface instead, producing X-ray pulsations. Of course, this model is oversimplified and has problems on both theoretical and observational levels. From a purely theoretical standpoint, between accretion and ejection we expect a "weak propeller" regime (Ustyugova et al. 2006), where the centrifugal barrier prevents accretion but the magnetically coupled material at the inner edge of the disk

\footnotetext{
11 As reported in Archibald et al. (2015), the pulsed fraction limit in the X-ray low mode $(2.4 \%$ at $95 \%$ confidence) is well below the observed pulsed fraction in the high mode $(8.1 \%)$.
}

does not have escape velocity and cannot be ejected. Furthermore, the X-ray luminosity even in the high modes is far below that predicted to be necessary for matter to reach the co-rotation radius; this can possibly be explained in terms of "trapped disk" scenarios (D'Angelo \& Spruit 2012), but it is unclear how ejection fits into these pictures. Moreover, MHD simulations of accretion in this regime show rapid switching between two modes but with a positive correlation: short episodes of accretion are accompanied by ejection, and these are interspersed with episodes where neither occurs; these episodes also occur on drastically shorter timescales (of the order of a few rotation cycles of the star) than we observe. Also, as noted previously, the rapid disappearance of the radio emission we report here poses challenges for jet-type models: how does the jet disappear so abruptly?

More nuanced explanations that do not invoke a "classical" jet are necessary. Baglio et al. (2016) found that the nearinfrared spectral energy distribution of PSR J1023+0038 does not show the excess expected from non-thermal jet emission. While the system shows optical linear polarization (Baglio et al. 2016; see also Hakala \& Kajava 2018), this is more likely due to Thomson scattering with electrons in the disk and not from synchrotron jet emission. Thus, there is no evidence in the optical or IR bands for a classical jet, although that would not be altogether surprising given that any jets at such low luminosities would be expected to have a spectral break frequency below the near-infrared band (e.g., Migliari et al. 2007).

An interpretation more consistent with the available observational evidence is that the flat-spectrum radio flux enhancements do not originate in a highly collimated outflow but are instead produced by particles accelerated by the propeller effect itself in the turbulent interface between the rapidly rotating pulsar magnetosphere and the accretion flow 
(Papitto \& Torres 2015). For an advection-dominated disk (as proposed by Campana et al. 2016), there would be sufficient pressure to push the innermost disk boundary to the co-rotation radius, allowing the activation of the propeller. This scenario is consistent with the close temporal alignment between the X-ray low mode and radio flux enhancements as the radio emission originates close to the pulsar.

Several interpretations for the multi-wavelength phenomenology of PSR J1023+0038 and the other accreting tMSPs have centered on the assumption of an active rotation-powered pulsar wind (see, e.g., Coti Zelati et al. 2014; Linares et al. 2014; Takata et al. 2014; Campana et al. 2016). The spin-down rate of PSR $\mathrm{J} 1023+0038$ has been measured to be $26.8 \pm 0.4 \%$ higher in the accretion state as compared to the radio pulsar state (Archibald et al. 2013; Jaodand et al. 2016). This is an indication that the pulsar spin-down still proceeds largely unaffected by the accretion inflow, meaning that even if the pulsar radio emission mechanism is disrupted in the accretion state, the bulk of the rotational energy loss is still in the form of a relativistic particle wind and Poynting flux.

Much of our insight into accreting neutron star systems and MSPs in particular has been gained from observations of highluminosity, that is, high accretion rate, states. In this regime, the power output of a rotation-powered pulsar relative to accretion power is vastly subdominant and its role in the observed properties is virtually negligible. In contrast, tMSPs reside in a regime where accretion and rotation power are comparable. In the case of PSR $\mathrm{J} 1023+0038$ in its present low-luminosity accreting state, the spin-down luminosity of $\dot{E} \approx 4 \times$ $10^{34} \mathrm{erg} \mathrm{s}^{-1}$ is only about an order of magnitude greater than the time-averaged X-ray luminosity, which is presumably powered mostly by accretion. Therefore, for an accretion efficiency of $G M / c^{2} \mathrm{R} \sim 0.1$, rotation and accretion power are comparable. In light of this, it is perhaps not surprising that the classical accretion inflow/jet outflow model cannot offer a satisfactory, self-consistent explanation of the observed X-ray/ radio behavior of PSR $\mathrm{J} 1023+0038$ due to the lack of a key ingredient, namely, an active, rapidly spinning rotationpowered pulsar that acts as an additional reservoir of energy for driving outflows. Recently, Parfrey et al. (2016, 2017) modeled the interaction of an active magnetosphere with a matter-dominated disk with a fixed velocity field, and finite thickness and conductivity. The simulations do indicate that for accreting MSPs the pulsar wind outflow may power the radio jets. However, they also predict a dramatic increase in spindown of the neutron star due to the opening of additional magnetic field lines, which is not observed in PSR J1023 +0038 (Jaodand et al. 2016), implying that in reality the magnetic field is much less efficient at coupling the star and disk. Parfrey \& Tchekhovskoy (2017) furthered these simulations by interacting a force-free magnetosphere with a fullMHD accretion disk in a general relativistic regime for the first time. The set of simulations produced four magnetic-fieldstrength-dependent regimes, including two that may account for the X-ray modes in tMSPs: (1) a regime in which the pulsar wind inhibits the accretion flow from moving past the light cylinder but drives shocks through it giving rise to X-ray emission, and (2) a regime in which the accreting matter is able to penetrate the light cylinder but is occasionally ejected by the rotating magnetic field. However, these simulations only span a real-time duration of $\sim 0.35 \mathrm{~s}$ (assuming a NS mass of $1.4 M_{\odot}$ ), in contrast to the observed X-ray mode-switching timescales of $\sim 10 \mathrm{~s}$. Although these simulations do not yet predict the detailed multi-wavelength behavior seen in PSR J1023+0038, they nevertheless represent important advances toward an understanding of accretion onto rotation-powered MSPs.

Under the assumption of an active pulsar, one possible interpretation of the anti-correlated X-ray/radio variability involves the rapid discharge of plasma from the pulsar magnetosphere or the inflation of a short-lived compact pulsar wind nebula. In this scenario, due to circumstances that remain to be understood, outflows from the pulsar are occasionally able to expand past the pulsar light cylinder in the orbital/ accretion disk plane and likely out to much larger distances above and below the disk. Presumably, accretion commences again once the rapidly expanding plasma cools sufficiently. The duration of an X-ray low mode/radio brightening interval probably depends on the initial conditions of the plasma ejection event.

If outflows from the pulsar are responsible for the observed $\mathrm{X}$-ray mode-switching and associated radio brightness enhancements a crucial question arises. What precipitates these episodic eruptions? The highly reproducible X-ray mode-switching behavior is not seen in other varieties of accreting systems. but is reminiscent of certain phenomenology associated with rotation-powered pulsars, such as radio mode-switching and nulling (Backer 1970; Timokhin 2010; Hermsen et al. 2013; Bilous et al. 2014; Mereghetti et al. 2016). While such phenomena likely occur in the absence of external drivers such as accreting material, they demonstrate that abrupt reconfiguration of the pulsar magnetosphere between discrete metastable states is possible. Although the disk-magnetosphere interaction is poorly understood, the relative energy budgets of accretion and spin-down, as well as the phenomenology, suggest that pulsar electrodynamics may govern the physical processes responsible for the peculiar switching behavior. The pulsar may also be responsible for the extraordinary stability of the high mode X-ray flux, which implies a near constant accretion rate onto the star over many years (Bogdanov et al. 2015; Jaodand et al. 2016), perhaps through an as-yet-unknown flow regulation mechanism imposed by the pulsar magnetosphere.

\section{Conclusions}

We have presented coordinated X-ray and radio observations of PSR J1023+0038 in its low-luminosity accreting state. For the first time, we conclusively establish the presence of strongly anti-correlated variability in the two bands. In particular, the radio flux density shows a rapid rise that commences at roughly the same time as the drop from a high to a low X-ray flux mode, peaking at most minutes later before beginning a decline back toward the "quiescent" level seen during the X-ray high mode. However, if this decline is not completed by the low $\rightarrow$ high mode transition, a relatively abrupt drop back to the quiescent level is seen around this time. This phenomenology implies a causal connection that is difficult to explain with any standard inflow/outflow models of accretion onto a compact object. The radio spectral evolution during a low mode (inverted early in the low mode, steep late in the low mode) is particularly difficult to explain in light of this causal connection, since it would be most easily interpreted as an expanding synchrotron-emitting plasma, whose causal connection to the accretion flow (as probed by the $\mathrm{X}$-ray) is difficult to fathom. 
We argue that the active rotation-powered pulsar plays a key role in the production of the anti-correlated X-ray/radio variability. The radio brightness enhancements accompanying $\mathrm{X}$-ray low modes might arise from plasma outflows driven by the active rotation-powered pulsar instead of the accretion inflow, while the mode-switching is the result of bistable reconfigurations of the pulsar magnetosphere, perhaps induced by the action of the accretion flow.

The simultaneous X-ray and radio data further reveal that, during the X-ray low modes, PSR J1023+0038 has a position in the $L_{X}-L_{R}$ diagram that makes it indistinguishable from a $\mathrm{BH}$ LMXB. This finding opens the possibility that some candidate low-luminosity BH LMXBs observed only for a short period of time may actually be tMSP impostors caught in an X-ray low mode, which implies that additional lines of observational evidence are needed to unambiguously establish the nature of the compact object (see, e.g., Bahramian et al. 2017, for an illustrative example).

We thank C. Heinke, E. Kuulkers, A. Papitto, and R. Wijnands for fruitful discussions at the Lorentz Center workshop "The Zoo of Accreting Compact Objects" held 2015 August 3-7, in particular for suggesting the use of the archival XMM-Newton RGS data. S.B. was funded in part by NASA Chandra Cycle 17 Guest Observer Program grant GO617034X awarded through Columbia University and issued by the Chandra X-ray Observatory Center, which is operated by the Smithsonian Astrophysical Observatory for and on behalf of NASA under contract NAS8-03060. A.T.D. and J.C.A.M.-J. are the recipients of Australian Research Council Future Fellowships (FT150100415 and FT140101082). A.M.A. is an NWO Veni fellow. J.W.T.H., A.P., and C.D. acknowledge support from NWO Vidi fellowships. J.W.T.H. and A.J. acknowledge support from the European Research Council under the European Union's Seventh Framework Programme (FP/2007-2013)/ERC Grant Agreement No. 337062. The National Radio Astronomy Observatory is a facility of the National Science Foundation operated under cooperative agreement by Associated Universities, Inc. This research has made use of the NASA Astrophysics Data System (ADS), the arXiv, and software provided by the Chandra X-ray Center (CXC) in the application package CIAO.

Facilities: CXO, VLA, XMM.

Software: CIAO (Fruscione et al. 2006), CASA (McMullin et al. 2007), SAS (Gabriel et al. 2004).

\section{ORCID iDs}

Slavko Bogdanov (i) https://orcid.org/0000-0002-9870-2742 Adam T. Deller (1) https://orcid.org/0000-0001-9434-3837 James C. A. Miller-Jones (1) https://orcid.org/0000-00033124-2814

Anne M. Archibald (ㄷ https://orcid.org/0000-0003-0638-3340 Jason W. T. Hessels (1) https://orcid.org/0000-00032317-1446

Amruta Jaodand (1) https://orcid.org/0000-0002-3850-6651

Alessandro Patruno (i) https://orcid.org/0000-0002-6459-0674

\section{References}

Archibald, A. M., Bogdanov, S., Patruno, A., et al. 2015, ApJ, 807, 62 Archibald, A. M., Kaspi, V. M., Hessels, J. W. T., et al. 2013, arXiv:1311.5161 Archibald, A. M., Stairs, I. H., Ransom, S. M., et al. 2009, Sci, 324, 1411 Backer, D. C. 1970, Natur, 228, 42

Baglio, M. C., D’Avanzo, P., Campana, S., et al. 2016, A\&A, 591, A101 Bahramian, A., Heinke, C. O., Tudor, V., et al. 2017, MNRAS, 467, 2199 Bilous, A. V., Hessels, J. W. T., Kondratiev, V. I., et al. 2014, A\&A, 572, A52 Blandford, R. D., \& Payne, D. G. 1982, MNRAS, 199, 883

Bogdanov, S., Archibald, A. M., Bassa, C., et al. 2015, ApJ, 806, 148 Bogdanov, S., \& Halpern, J. P. 2015, ApJL, 803, L27

Campana, S., Coti Zelati, F., Papitto, A., et al. 2016, A\&A, 594, A31 Chomiuk, L., Strader, J., Maccarone, T. J., et al. 2013, ApJ, 777, 69 Coti Zelati, F., Baglio, M. C., Campana, S., et al. 2014, MNRAS, 444, 1783 D'Angelo, C. R., \& Spruit, H. C. 2012, MNRAS, 420, 416

de Martino, D., Belloni, T., Falanga, M., et al. 2013, A\&A, 550, A89

Deller, A. T., Archibald, A. M., Brisken, W. F., et al. 2012, ApJL, 756, L25

Deller, A. T., Moldon, J., Miller-Jones, J. C. A., et al. 2015, ApJ, 809, 13

Fender, R. P., Belloni, T. M., \& Gallo, E. 2004, MNRAS, 355, 1105

Fender, R. P., Pooley, G. G., Durouchoux, P., Tilanus, R. P. J., \& Brocksopp, C. 2000, MNRAS, 312, 853

Fruscione, A., McDowell, J. C., Allen, G. E., et al. 2006, Proc. SPIE, 6270, $62701 \mathrm{~V}$

Gabriel, C., Denby, M., Fyfe, D. J., et al. 2004, in ASP Conf. Ser. 314 , Astronomical Data Analysis Software and Systems (ADASS) XIII, ed. F. Ochsenbein, M. G. Allen, \& D. Egret (San Francisco, CA: ASP), 759

Gallo, E., Fender, R. P., \& Hynes, R. I. 2005, MNRAS, 356, 1017

Gallo, E., Miller-Jones, J. C. A., Russell, D. M., et al. 2014, MNRAS, 445, 290

Gusinskaia, N. V., Deller, A. T., Hessels, J. W. T., et al. 2017, MNRAS, 470,1871

Hakala, P., \& Kajava, J. J. E. 2018, MNRAS, 474, 3297

Hermsen, W., Hessels, J. W. T., Kuiper, L., et al. 2013, Sci, 339, 436

Hill, A. B., Szostek, A., Corbel, S., et al. 2011, MNRAS, 415, 235

Jaodand, A., Archibald, A. M., Hessels, J. W. T., et al. 2016, ApJ, 830, 122

Linares, M., Bahramian, A., Heinke, C., et al. 2014, MNRAS, 438, 251

McConnell, O., Callanan, P. J., Kennedy, M., et al. 2015, MNRAS, 451, 3468

McMullin, J. P., Waters, B., Schiebel, D., Young, W., \& Golap, K. 2007, in ASP Conf. Ser. 376, Astronomical Data Analysis Software and Systems XVI, ed. R. A. Shaw, F. Hill, \& D. J. Bell (San Francisco, CA: ASP), 127 Mereghetti, S., Kuiper, L., Tiengo, A., et al. 2016, ApJ, 831, 21

Migliari, S., \& Fender, R. P. 2006, MNRAS, 366, 79

Migliari, S., Miller-Jones, J. C. A., \& Russell, D. M. 2011, MNRAS, 415, 2407

Migliari, S., Tomsick, J. A., Maccarone, T. J., et al. 2006, ApJL, 643, L41

Migliari, S., Tomsick, J. A., Markoff, S., et al. 2007, ApJ, 670, 610

Migliari, S., Tomsick, J. A., Miller-Jones, J. C. A., et al. 2010, ApJ, 710, 117 Miller-Jones, J. C. A., Strader, J., Heinke, C. O., et al. 2015, MNRAS, 453, 3918

Papitto, A., Ferrigno, C., Bozzo, E., et al. 2013, Natur, 501, 517

Papitto, A., \& Torres, D. F. 2015, ApJ, 807, 33

Parfrey, K., Spitkovsky, A., \& Beloborodov, A. M. 2016, ApJ, 822, 33

Parfrey, K., Spitkovsky, A., \& Beloborodov, A. M. 2017, MNRAS, 469, 3656

Parfrey, K., \& Tchekhovskoy, A. 2017, ApJL, 851, L34

Patruno, A., Archibald, A. M., Hessels, J. W. T., et al. 2014, ApJL, 781, L3

Patruno, A., \& Watts, A. L. 2012, arXiv:1206.2727

Stappers, B. W., Archibald, A. M., Hessels, J. W. T., et al. 2014, ApJ, 790, 39

Stirling, A. M., Spencer, R. E., de la Force, C. J., et al. 2001, MNRAS, 327, 1273

Strader, J., Chomiuk, L., Maccarone, T. J., Miller-Jones, J. C. A., \& Seth, A. C. 2012, Natur, 490, 71

Takata, J., Li, K. L., Leung, G. C. K., et al. 2014, ApJ, 785, 131

Tendulkar, S. P., Yang, C., An, H., et al. 2014, ApJ, 791, 77

Tetarenko, A. J., Bahramian, A., Sivakoff, G. R., et al. 2016, MNRAS, 460, 345 Thorstensen, J. R., \& Armstrong, E. 2005, AJ, 130, 759

Timokhin, A. N. 2010, MNRAS, 408, L41

Tudor, V., Miller-Jones, J. C. A., Patruno, A., et al. 2017, MNRAS, 470, 324

Ustyugova, G. V., Koldoba, A. V., Romanova, M. M., \& Lovelace, R. V. E. 2006, ApJ, 646, 304

Vadawale, S. V., Rao, A. R., Naik, S., et al. 2003, ApJ, 597, 1023

Wang, Z., Archibald, A. M., Thorstensen, J. R., et al. 2009, ApJ, 703, 2017 